\title{
ASYMPTOTIC BEHAVIOR OF NONEXPANSIVE SEQUENCES AND MEAN POINTS
}

\author{
JONG SOO JUNG AND JONG SEO PARK
}

(Communicated by Palle E. T. Jorgensen)

\begin{abstract}
Let $E$ be a real Banach space with norm $\|\cdot\|$ and let $\left\{x_{n}\right\}_{n \geq 0}$ be a nonexpansive sequence in $E$ (i.e., $\left\|x_{i+1}-x_{j+1}\right\| \leq\left\|x_{i}-x_{j}\right\|$ for all $i, j \geq 0$ ). Let $K=\bigcap_{n=1}^{\infty} \overline{c o}\left\{\left\{x_{i}-x_{i-1}\right\}_{i \geq n}\right\}$. We deal with the mean point of $\left\{\frac{x_{n}}{n}\right\}$ concerning a Banach limit. We show that if $E$ is reflexive and $d=d(0, K)$, then $d=d\left(0, \overline{c o}\left\{\frac{x_{n}-x_{0}}{n}\right\}\right)$ and there exists a unique point $z_{0}$ with $\left\|z_{0}\right\|=d$ such that $z_{0} \in \overline{c o}\left\{\frac{x_{n}-x_{0}}{n}\right\}$. This result is applied to obtain the weak and strong convergence of $\left\{\frac{x_{n}}{n}\right\}$.
\end{abstract}

\section{INTRODUCTION}

Let $E$ be a real Banach space with norm $\|\cdot\|$ and let $\left\{x_{n}\right\}_{n \geq 0}$ be a nonexpansive sequence in $E$ (i.e., $\left\|x_{i+1}-x_{j+1}\right\| \leq\left\|x_{i}-x_{j}\right\|$ for all $i, j \geq 0$ ). Recently, Djafari Rouhani [2] obtained an interesting result on the weak convergence of $\left\{\frac{x_{n}}{n}\right\}$ under the assumption that $E$ is reflexive and strictly convex.

In this paper, we deal with his result without the assumption of strict convexity of $E$. That is, instead of the weak limit of $\left\{\frac{x_{n}}{n}\right\}$, we deal with the mean point of $\left\{\frac{x_{n}}{n}\right\}$ concerning a Banach limit under the assumption that $E$ is reflexive. Using the mean point, we obtain the weak convergence of $\left\{\frac{x_{n}}{n}\right\}$, in which case $E$ is reflexive and strictly convex. Also we obtain the strong convergence of $\left\{\frac{x_{n}}{n}\right\}$, in which case $E^{*}$ has a Fréchet differentiable norm. Our results improve and extend the corresponding results in $[5,6,7,8,9,10]$ as in [2].

\section{Preliminaries}

Let $E$ be a real Banach space with norm $\|\cdot\|$ and dual $\left(E^{*},\|\cdot\|\right)$. The duality pairing between $E$ and $E^{*}$ will be denoted by $(\cdot, \cdot)$. The duality mapping $J$ from $E$ into the family of nonempty closed convex subsets of $E^{*}$ is defined by

$$
J(x)=\left\{x^{*} \in E^{*}:\left(x, x^{*}\right)=\|x\|^{2}=\left\|x^{*}\right\|^{2}\right\} .
$$

Received by the editors March 24, 1994 and, in revised form, August 22, 1994.

1991 Mathematics Subject Classification. Primary 47H09.

Key words and phrases. Asymptotic behavior, Banach limit, mean point, nonexpansive sequence.

This research was supported by the Korea Science and Engineering Foundation, project number 941-0100-035-2. 
Note that we have for $x, y \in E$ and $j \in J(x)$,

$$
(x-y, j)=\|x\|^{2}-(y, j) \geq\|x\|^{2}-\frac{1}{2}\left(\|y\|^{2}+\|j\|^{2}\right)=\frac{1}{2}\left(\|x\|^{2}-\|y\|^{2}\right) .
$$

We recall that if $E$ is reflexive and strictly convex and $K$ is a nonempty closed convex subset of $E$, the nearest point projection mapping $P_{K}$ of $E$ onto $K$ is well defined, i.e., $K$ is a Chebyshev set (see $[1,4])$.

We say that the sequence $\left\{x_{n}\right\}_{n \geq 0}$ is nonexpansive if $\left\|x_{i+1}-x_{j+1}\right\| \leq\left\|x_{i}-x_{j}\right\|$ for all $i, j \geq 0$.

Let $\mu$ be a mean on integers $\mathrm{N}$, i.e., a continuous linear functional on $\ell^{\infty}$ satisfying $\|\mu\|=1=\mu(1)$. Then we know that $\mu$ is a mean on $\mathrm{N}$ if and only if

$$
\inf \left\{a_{n}: n \in \mathrm{N}\right\} \leq \mu(a) \leq \sup \left\{a_{n}: n \in \mathrm{N}\right\}
$$

for every $a=\left(a_{1}, a_{2}, \cdots\right) \in \ell^{\infty}$. According to time and circumstances, we use $\mu_{n}\left(a_{n}\right)$ instead of $\mu(a)$. A mean $\mu$ on $\mathrm{N}$ is called a Banach limit if

$$
\mu_{n}\left(a_{n}\right)=\mu_{n}\left(a_{n+1}\right)
$$

for every $a=\left(a_{1}, a_{2}, \cdots\right) \in \ell^{\infty}$. Using the Hahn-Banach theorem, we can prove the existence of a Banach limit. We know that if $\mu$ is a Banach limit, then

$$
\liminf _{n \rightarrow \infty} a_{n} \leq \mu_{n}\left(a_{n}\right) \leq \limsup _{n \rightarrow \infty} a_{n}
$$

for every $a=\left(a_{1}, a_{2}, \cdots\right) \in \ell^{\infty}$.

Let $E$ be a reflexive Banach space and let $\left\{x_{n}\right\}$ be a bounded sequence in $E$. Then, for a Banach limit $\mu$, we can obtain a point $x_{0}$ in $E$ such that

$$
\mu_{n}\left(x_{n}, x^{*}\right)=\left(x_{0}, x^{*}\right)
$$

for all $x^{*} \in E^{*}$. In fact, the function $\mu_{n}\left(x_{n}, x^{*}\right)$ is linear in $x^{*}$. Further, since

$$
\left.\left|\mu_{n}\left(x_{n}, x^{*}\right)\right| \leq \sup _{n}\left\|x_{n}\right\|\right) \cdot\left\|x^{*}\right\|,
$$

the function $\mu_{n}\left(x_{n}, x^{*}\right)$ is also bounded in $x^{*}$. So, we have $x_{0}^{* *} \in E^{* *}$ such that $\mu_{n}\left(x_{n}, x^{*}\right)=\left(x_{0}^{* *}, x^{*}\right)$ for every $x^{*} \in E^{*}$. Since $E$ is reflexive, we obtain $x_{0} \in E$ such that $\mu_{n}\left(x_{n}, x^{*}\right)=\left(x_{0}, x^{*}\right)$ for all $x^{*} \in E^{*}$. This point $x_{0}$ is called a mean point of $\left\{x_{n}\right\}$ concerning $\mu$. We also know that this mean point $x_{0}$ is contained in $\bigcap_{n \geq 1}^{\infty} \overline{c o}\left\{x_{n}\right\}$. In fact, if not, there exists $n_{0} \in \mathrm{N}$ such that $x_{0} \notin \overline{c o}\left\{x_{n}: n \geq n_{0}\right\}$. By separation theorem, we obtain a point $x^{*} \in E^{*}$ such that

$$
\left(x_{0}, x^{*}\right)<\inf \left\{\left(z, x^{*}\right): z \in \overline{c o}\left\{x_{n}: n \geq n_{0}\right\}\right\} .
$$

So, we have

$$
\begin{aligned}
\mu_{n}\left(x_{n}, x^{*}\right)=\left(x_{0}, x^{*}\right) & <\inf \left\{\left(x_{n}, x^{*}\right): n \geq n_{0}\right\} \\
& \leq \mu_{n}\left\{\left(x_{n}, x^{*}\right): n \geq n_{0}\right\}=\mu_{n}\left(x_{n}, x^{*}\right) .
\end{aligned}
$$

This is a contradiction. For these facts, see [11].

Let $S=\{x \in E:\|x\|=1\}$. Then the norm of $E$ is Fréchet differentiable if for each $x \in S$, the limit

$$
\lim _{t \rightarrow 0} \frac{\|x+t y\|-\|x\|}{t}
$$

exists uniformly for each $y \in S$. The following lemma is well known (cf. [3]). 
Lemma 2.1. $E^{*}$ has a Fréchet differentiable norm if and only if $E$ is reflexive and strictly convex, and has the following property : if the weak $\lim _{n \rightarrow \infty} x_{n}=x$ and $\lim _{n \rightarrow \infty}\left\|x_{n}\right\|=\|x\|$ for a sequence $\left\{x_{n}\right\}$ in $E$, then $\left\{x_{n}\right\}$ converges strongly to $x$.

Finally, let $D$ be a subset of $E$. Then we denote the closure of $D$ by $\bar{D}$ and the closed convex hull of $D$ by $\overline{c o} D$, respectively. We also denote its distance from a point $x$ in $E$ by $d(x, D)=\inf _{y \in D}\|x-y\|$.

\section{Main Result}

In this section, for a nonexpansive sequence $\left\{x_{n}\right\}$ in $E$, we study the mean point of $\left\{\frac{x_{n}}{n}\right\}$ concerning a Banach limit.

We begin with the known result which will play a crucial role in our result.

Lemma 3.1 [2]. Let $E$ be a Banach space and let $\left\{x_{n}\right\}$ be a nonexpansive sequence in $E$. Then

$$
\lim _{n \rightarrow \infty}\left\|\frac{x_{n}}{n}\right\| \text { exists and } \lim _{n \rightarrow \infty}\left\|\frac{x_{n}}{n}\right\|=\inf _{n \geq 1}\left\|\frac{x_{n}-x_{0}}{n}\right\| .
$$

The following result is essentially in spirit of Djafari Rouhani [2]. For completeness, we give the proof.

Lemma 3.2. Let $E$ be a reflexive Banach space and let $\left\{x_{n}\right\}$ be a nonexpansive sequence in $E$. Let

$$
K=\bigcap_{n=1}^{\infty} \overline{c o}\left\{\left\{x_{i}-x_{i-1}\right\}_{i \geq n}\right\}
$$

Then $\lim _{n \rightarrow \infty}\left\|\frac{x_{n}}{n}\right\|=d(0, K)=\inf _{n \geq 1}\left\|\frac{x_{n}-x_{0}}{n}\right\|$.

Proof. Let $k \geq 1$ be fixed and $j_{n} \in J\left(x_{n}-x_{k-1}\right)$ for $n \geq k$. Then we have for $n \geq k \geq 1$

$$
\begin{aligned}
\left(x_{k}-x_{k-1}, j_{n}\right) & \geq \frac{1}{2}\left\|x_{n}-x_{k-1}\right\|^{2}-\frac{1}{2}\left\|x_{n}-x_{k}\right\|^{2} \\
& \geq \frac{1}{2}\left\|x_{n}-x_{k-1}\right\|^{2}-\frac{1}{2}\left\|x_{n-1}-x_{k-1}\right\|^{2} .
\end{aligned}
$$

Hence we obtain

$$
\frac{2}{n^{2}}\left(x_{k}-x_{k-1}, \sum_{i=k}^{n} j_{i}\right) \geq\left\|\frac{x_{n}-x_{k-1}}{n}\right\|^{2} .
$$

Let $S_{n}=\frac{2}{n^{2}} \sum_{i=k}^{n} j_{i}$ for $n \geq k$. Then we have

$$
\left\|S_{n}\right\| \leq \frac{2}{n^{2}} \sum_{i=k}^{n}\left\|x_{i}-x_{k-1}\right\|=\frac{2}{n^{2}} \sum_{i=k}^{n} i\left\|\frac{x_{i}-x_{k-1}}{i}\right\|
$$

and so $\left\{S_{n}\right\}$ is bounded since $\left\{\frac{x_{n}}{n}\right\}$ is bounded by Lemma 3.1. Thus from the weak-star compactness of the closed unit ball of $E^{*}$, it follows that the sequence 
$\left\{S_{n}\right\}$ has a weak-star cluster point $j \in E^{*}$ (obviously independent of $k \geq 1$ ). Now by Lemma 3.1 and (3.1), we obtain

$$
\left(x_{k}-x_{k-1}, j\right) \geq \lim _{n \rightarrow \infty}\left\|\frac{x_{n}}{n}\right\|^{2}
$$

for $k \geq 1$ and hence

$$
\left(\frac{x_{n}-x_{0}}{n}, j\right) \geq \lim _{n \rightarrow \infty}\left\|\frac{x_{n}}{n}\right\|^{2}
$$

for all $n \geq 1$. We also have

$$
\begin{aligned}
\|j\| & \leq \liminf _{n \rightarrow \infty}\left\|S_{n}\right\| \\
& \leq \limsup _{n \rightarrow \infty} \frac{2}{n^{2}} \sum_{i=k}^{n} i\left\|\frac{x_{i}-x_{k-1}}{i}\right\|=\lim _{n \rightarrow \infty}\left\|\frac{x_{n}}{n}\right\|
\end{aligned}
$$

and so

$$
\left(x_{k}-x_{k-1}, j\right) \geq \lim _{n \rightarrow \infty}\left\|\frac{x_{n}}{n}\right\|^{2} \geq\|j\|^{2}
$$

for all $k \geq 1$. So, for any $z \in \overline{c o}\left\{\left\{x_{i+1}-x_{i}\right\}_{i \geq 0}\right\}$,

$$
\begin{aligned}
\frac{1}{2} \lim _{n \rightarrow \infty}\left\|\frac{x_{n}}{n}\right\|^{2}+\frac{1}{2}\|z\|^{2} & \geq \frac{1}{2}\|j\|^{2}+\frac{1}{2}\|z\|^{2} \\
& \geq(z, j) \geq \lim _{n \rightarrow \infty}\left\|\frac{x_{n}}{n}\right\|^{2} \geq\|j\|^{2} .
\end{aligned}
$$

Since $K \subset \overline{c o}\left\{\left\{x_{i+1}-x_{i}\right\}_{i \geq 0}\right\}$, it follows from (3.3) that

$$
\|j\| \leq \lim _{n \rightarrow \infty}\left\|\frac{x_{n}}{n}\right\| \leq \inf _{z \in K}\|z\|=d(0, K) .
$$

On the other hand, since $\left\{\frac{x_{n}}{n}\right\}$ is bounded and $E$ is reflexive, $\left\{\frac{x_{n}-x_{0}}{n}\right\}$ contains a weakly convergent subsequence $\left\{\frac{x_{n_{l}}-x_{0}}{n_{l}}\right\}$. Let $\left\{\frac{x_{n_{l}}-x_{0}}{n_{l}}\right\}$ converge weakly to $q \in K$. Then we have

$$
\|q\| \leq \liminf _{l \rightarrow \infty}\left\|\frac{x_{n_{l}}-x_{0}}{n_{l}}\right\|=\lim _{n \rightarrow \infty}\left\|\frac{x_{n}}{n}\right\|
$$

and hence $\lim _{n \rightarrow \infty}\left\|\frac{x_{n}}{n}\right\|=d(0, K)$. This completes the proof.

Now, using Lemmas 3.1 and 3.2, we obtain the main result.

Theorem 3.3. Let $E$ be a reflexive Banach space and let $\left\{x_{n}\right\}$ be a nonexpansive sequence in E. Let

$$
K=\bigcap_{n=1}^{\infty} \overline{c o}\left\{\left\{x_{i}-x_{i-1}\right\}_{i \geq n}\right\}
$$

and $d=d(0, K)$. Then $d=d\left(0, \overline{c o}\left\{\frac{x_{n}-x_{0}}{n}\right\}\right)$ and there exists a point $z_{0}$ with $\left\|z_{0}\right\|=$ $d$ such that $z_{0} \in \overline{c o}\left\{\frac{x_{n}-x_{0}}{n}\right\}$.

Proof. Since $\lim _{n \rightarrow \infty}\left\|\frac{x_{n}}{n}\right\|=\lim _{n \rightarrow \infty}\left\|\frac{x_{n}-x_{0}}{n}\right\|=d$ by Lemma 3.2, we may assume that $\left\{\frac{x_{n}-x_{0}}{n}\right\}$ is bounded. So, it follows from reflexivity of $E$ that for a Banach limit $\mu$, there exists $z_{0} \in \overline{c o}\left\{\frac{x_{n}-x_{0}}{n}\right\}$ such that

$$
\mu_{n}\left(\frac{x_{n}-x_{0}}{n}, x^{*}\right)=\left(z_{0}, x^{*}\right)
$$


for every $x^{*} \in E^{*}$. For $j_{0} \in J\left(z_{0}\right)$, where $J$ is the duality mapping of $E$, we have

$$
\begin{aligned}
\left\|z_{0}\right\|^{2}=\left(z_{0}, j_{0}\right) & =\mu_{n}\left(\frac{x_{n}-x_{0}}{n}, j_{0}\right) \\
& \leq \mu_{n}\left(\left\|\frac{x_{n}-x_{0}}{n}\right\|\right) \cdot\left\|j_{0}\right\|=d \cdot\left\|j_{0}\right\|=d \cdot\left\|z_{0}\right\|,
\end{aligned}
$$

and hence $\left\|z_{0}\right\| \leq d$. On the other hand, by the proof of Lemma 3.2 and (3.2), there exists a functional $j \in E^{*}$ with $\|j\| \leq d$ such that

$$
\left(\frac{x_{n}-x_{0}}{n}, j\right) \geq d^{2}
$$

for all $n \geq 1$. Hence we have $\left(z_{0}, j\right) \geq d^{2}$. Since $\|j\| \leq d$, we obtain

$$
d^{2} \geq\left\|z_{0}\right\| \cdot\|j\| \geq\left(z_{0}, j\right) \geq d^{2}
$$

and hence $\left\|z_{0}\right\|=\|j\|=d$. It also follows from (3.5) that $(z, j) \geq d^{2}$ for every $z \in \overline{c o}\left\{\frac{x_{n}-x_{0}}{n}\right\}$ and so

$$
\|z\| \cdot d=\|z\| \cdot\|j\| \geq(z, j) \geq d^{2} .
$$

Hence we have $\|z\| \geq d$ for every $z \in \overline{c o}\left\{\frac{x_{n}-x_{0}}{n}\right\}$. Then we obtain

$$
d=d\left(0, \overline{c o}\left\{\frac{x_{n}-x_{0}}{n}\right\}\right) \text {. }
$$

Let $w_{0}$ be another point satisfying (3.4). Then for $j \in J\left(z_{0}-w_{0}\right)$, we have

$$
\left\|z_{0}-w_{0}\right\|^{2}=\left(z_{0}-w_{0}, j\right)=\mu_{n}\left(\frac{x_{n}-x_{0}}{n}-\frac{x_{n}-x_{0}}{n}, j\right)=0,
$$

and hence $z_{0}=w_{0}$. This completes the proof.

Corollary 3.4. With the same assumptions as in Theorem 3.3, we have the following :

(i) If $E$ is strictly convex, then the weak $\lim _{n \rightarrow \infty} \frac{x_{n}}{n}$ exists and coincides with $P_{K} 0$ with $\left\|P_{K} 0\right\|=d$.

(ii) If $E^{*}$ has a Fréchet differentiable norm, then the strong $\lim _{n \rightarrow \infty} \frac{x_{n}}{n}$ exists and coincides with $P_{K} 0$.

Proof. (i) Since a reflexive Banach space $E$ is strictly convex, the set

$$
\left\{z \in \overline{c o}\left\{\frac{x_{n}-x_{0}}{n}\right\}:\|z\|=d\right\}
$$

consists of exactly one point and $d(0, K)=\left\|P_{K} 0\right\|$. This point equals $z_{0}$ in Theorem 3.3. Let $\left\{\frac{x_{n_{l}}}{n_{l}}\right\}$ be a subsequence of $\left\{\frac{x_{n}}{n}\right\}$ such that $\left\{\frac{x_{n_{l}}}{n_{l}}\right\}$ converges weakly to $p \in K$. Then since

$$
\|p\| \leq \liminf _{l}\left\|\frac{x_{n_{l}}}{n_{l}}\right\|=\lim _{n \rightarrow \infty}\left\|\frac{x_{n}}{n}\right\|=\left\|P_{K} 0\right\|,
$$

we have $p=z_{0}=P_{K} 0$. This implies that $\left\{\frac{x_{n}}{n}\right\}$ converges weakly to $P_{K} 0$, which completes the proof.

(ii) It follows from Lemma 2.1 that $\left\{\frac{x_{n}}{n}\right\}$ converges strongly to $P_{K} 0$.

Remark. (1) Since our study is equivalent to the study of the asymptotic behavior of the sequence $\left\{\frac{T^{n} x}{n}\right\}$ in $E$, where $T$ is a nonexpansive mapping from an arbitrary subset $C$ of $E$ into itself and $x \in C$, Theorem 3.3 is an improvement of Theorem 5 in [11].

(2) Corollary 3.4 is of interest in view of using the mean point. Compare this with Corollary 3.2 in [2].

(3) Corollary 3.4 also contains the previous corresponding results in $[5,6,7,8$, $9,10]$. 


\section{REFERENCES}

1. V. Barbu, Nonlinear semigroups and differential equations in Banach spaces, Editura Acad. R. S. R., Bucutesti, 1976. MR 52:11666

2. B. Djafari Rouhani, Asymptotic behavior of unbounded nonexpansive sequences in Banach spaces, Proc. Amer. Math. Soc. 117 (1993), 951-956. MR 93e:47073

3. K. Fan and I. Glicksberg, Some geometric properties of the spheres in a normed linear space, Duke Math. J. 25 (1958), 553-568. MR 20:5421

4. K. Goebel and S. Reich, Uniform convexity hyperbolic geometry and nonexpansive mappings, Dekker, New York and Basel, 1984. MR 86d:58012

5. E. Kohlberg and A. Neyman, Asymptotic behavior of nonexpansive mappings in uniformly convex Banach spaces, Amer. Math. Monthly 88 (1981), 698-700. MR 83c:47077

6. _ Asymptotic behavior of nonexpansive mappings in normed linear spaces, Israel $\mathrm{J}$. Math. 38 (1981), 269-275. MR 83g:47056

7. A. Pazy, Asymptotic behavior of contractions in Hilbert space, Israel J. Math. 9 (1971), 235240. MR 43:7988

8. A. T. Plant and S. Reich, The asymptotics of nonexpansive iterations, J. Funct. Anal. 54 (1983), 308-319. MR 85a:47055

9. S. Reich, On the asymptotic behavior of nonlinear semigroups and the range of accretive operators, J. Math. Anal. Appl. 78 (1981), 113-126. MR 82c:47066

10. $\quad$ On the asymptotic behavior of nonlinear semigroups and the range of accretive operators II, J. Math. Anal. Appl. 87 (1982), 134-146. MR 83e:47059

11. W. Takahashi, The asymptotic behavior of nonlinear semigroups and invariant means, J. Math. Anal. Appl. 109 (1985), 130-139. MR 86k:47045

Department of Mathematics, Dong-A University, Pusan 604-714, Korea

E-mail address: jungjs@seanghak.donga.ac.kr.

Department of Mathematics, Graduate School, Dong-A University, Pusan 604-714, KOREA 